\title{
Copper Homeostasis in Aspergillus fumigatus: Opportunities for Therapeutic Development
}

\author{
Jinxing Song ${ }^{*}$, Rongpeng $\mathrm{Li}^{\star}$ and Jihong Jiang* \\ The Key Laboratory of Biotechnology for Medicinal Plants of Jiangsu Province and School of Life Science, Jiangsu Normal \\ University, Xuzhou, China
}

\section{OPEN ACCESS}

Edited by:

Miguel Cacho Teixeira,

Universidade de Lisboa,

Portugal

Reviewed by:

Nir Osherov,

Tel Aviv University, Israel

Daisuke Hagiwara,

University of Tsukuba, Japan

${ }^{*}$ Correspondence: Jinxing Song

zdsongjinxing@jsnu.edu.cn

Rongpeng $\mathrm{Li}$

lirongpeng@jsnu.edu.cn

Jihong Jiang

jhjiang@jsnu.edu.cn

Specialty section:

This article was submitted to Antimicrobials, Resistance and

Chemotherapy,

a section of the journal

Frontiers in Microbiology

Received: 04 January 2019

Accepted: 26 March 2019

Published: 12 April 2019

Citation:

Song J, Li R and Jiang J (2019) Copper Homeostasis in Aspergillus

fumigatus: Opportunities for

Therapeutic Development.

Front. Microbiol. 10:774.

doi: 10.3389/fmicb.2019.00774
Aspergillus fumigatus can cause severe invasive aspergillosis in immunocompromised individuals. Copper, an essential but potentially toxic trace element for $A$. fumigatus, plays a critical role at the host-pathogen axis during infection. Accumulating evidence demonstrates that the host utilizes copper compartmentalization within macrophages to combat $A$. fumigatus infection. To survive under host-imposed copper toxicity, $A$. fumigatus has evolved sophisticated machinery to regulate copper homeostasis. Thus, targeting molecular pathways critical for copper homeostasis regulation provides an opportunity to improve therapeutic options for aspergillosis caused by $A$. fumigatus. In this review, we describe the copper homeostatic mechanisms by which $A$. fumigatus acquires and controls copper levels and explores the responses of the pathogen to alter copper levels in the host. Finally, we discuss the regulatory mechanisms of copper homeostasis that could be targeted for antifungal drug development.

Keywords: Aspergillus fumigatus, copper homeostasis, antifungal therapeutic target, copper transporters, copper transcription factor

\section{INTRODUCTION}

Aspergillus fumigatus is one of the most prevalent airborne fungal pathogens, causing severe invasive aspergillosis in immunocompromised individuals (Brown et al., 2012a; Kwon-Chung and Sugui, 2013). Current therapies used to combat A. fumigatus that have limited efficacy. Only three major classes of antifungal drugs (polyenes, azoles, and echinocandins) are used in the clinic (Xie et al., 2014). However, the efficacy of these antifungal drugs is limited, and A. fumigatus and other pathogenic fungi are either intrinsically resistant or have developed resistance over time (Shapiro et al., 2011; Brown et al., 2012b; Robbins et al., 2017). Accordingly, new strategies and molecules to increase efficacy against pathogenic targets must be developed.

Copper is essential for many forms of eukaryotic life, but it can also be toxic due in part to its ability to generate reactive oxygen species (ROS) (Fridovich, 1983; Macomber and Imlay, 2009; Nevitt et al., 2012). This duality provides a promising measure for antifungal therapy development. Copper has historically been used to control fungal and bacterial growth in healthcare settings (Casey et al., 2010; Lazary et al., 2014). It has become apparent that hosts use copper to fight A. fumigatus infection, and A. fumigatus has also evolved some mechanisms to resist coppermediated toxicity by regulating copper homeostasis (Kusuya et al., 2017; Wiemann et al., 2017; Cai et al., 2018; Park et al., 2018). Thus, copper plays an important role at the host-A. fumigatus axis, and 
a detailed understanding of copper homeostasis regulation in A. fumigatus can lead to new strategies for antifungal drug development. Therefore, it is worthwhile to provide an updated summary of A. fumigatus copper homeostasis, and highlight regulators of copper homeostasis as promising therapeutic targets for antifungal drug development.

\section{CORE COMPONENTS OF THE COPPER HOMEOSTASIS SYSTEM IN A. FUMIGATUS}

\section{Copper Transporters Are Involved in Copper Uptake}

A copper uptake system at the fungal plasma membrane facilitates the import of copper across the plasma membrane and into the cytosol. Currently, much can be learned regarding the copper uptake system in A. fumigatus through examination of more well-studied systems in yeast (Smith et al., 2017). In the model yeast Saccharomyces cerevisiae, the copper uptake system consists of a low-affinity transporter protein (Fet4) and three high affinity transporter proteins, namely Ctr1, Ctr2, and Ctr3 (Dancis et al., 1994; Hassett et al., 2000; Pena et al., 2000; Rees and Thiele, 2007). Ctr1 and Ctr3, both of which are localized to the plasma membrane, transport copper into the cytosol. Meanwhile, Ctr2, which is localized to the vacuole membrane, pumps copper into the cytosol (Rees and Thiele, 2007). As Ctr family members are highly conserved across fungal species, A. fumigatus also encodes four putative Ctr family members designated as CtrA1, CtrA2, CtrB, and CtrC (Figure 1; Park et al., 2014), and each Ctr member has at least two transmembrane domains, with a characteristic Met-X ( $n=1-5)$-Met motif that is necessary for copper transport. Among the four transporters, both CtrA2 and CtrC have been identified as high-affinity copper transporters that are responsible for copper uptake from low-copper environments (Park et al., 2014).

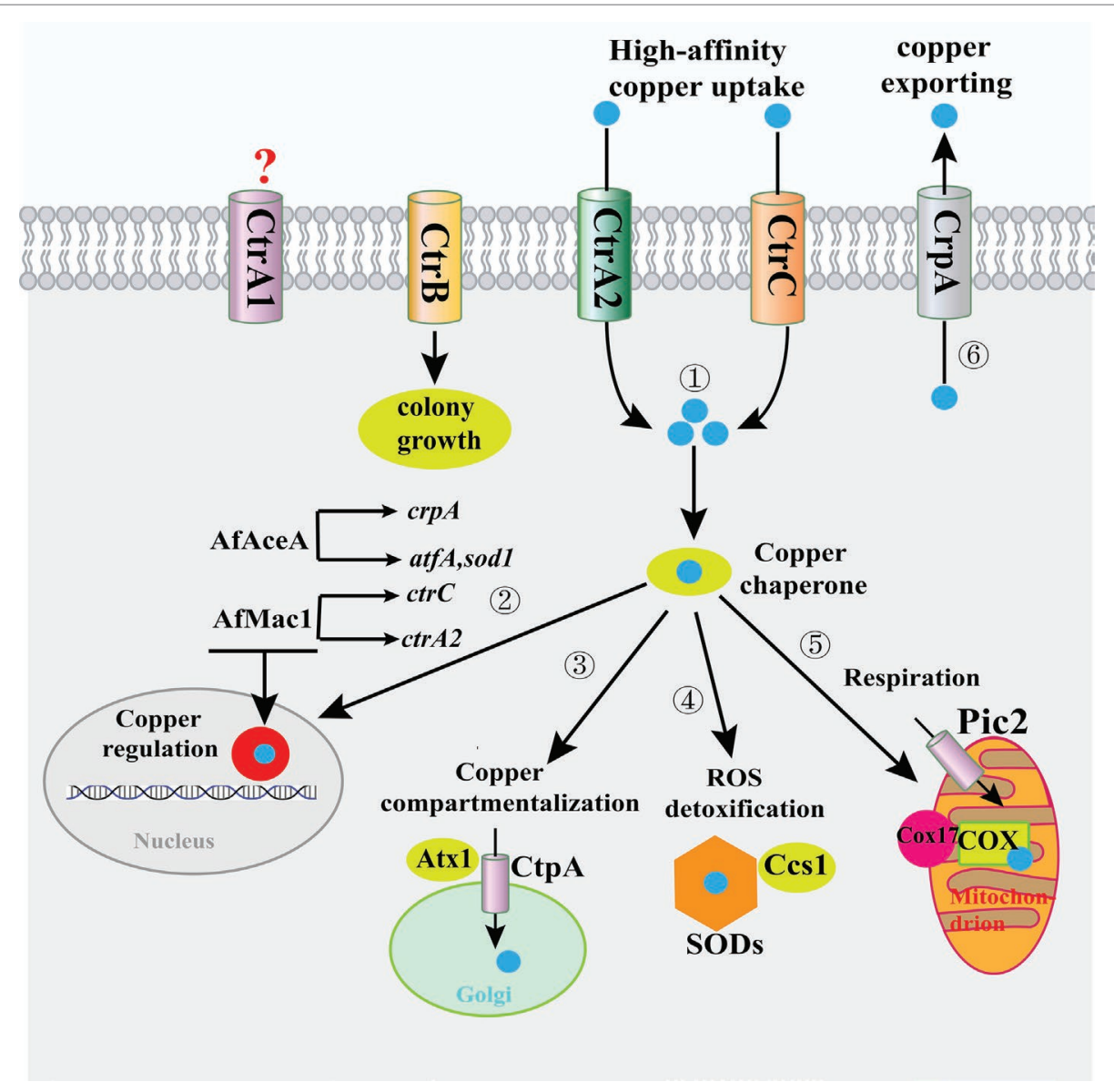

FIGURE 1 | Copper homeostasis mechanisms in A. fumigatus. (1) High-affinity copper transporters at the plasma membrane participate in copper (b/ue spheres) transport from the extracellular environment to the intracellular environment. (2) In the cytoplasm, copper is bound by copper chaperones that facilitate the delivery of copper to the nucleus for copper homeostasis regulation; AfMac1 induces the ctr $C$ and ctrA2 genes to respond to low copper, and AfAceA induces the expression of the copper extrusion pump CrpA, as well as the ROS detoxification proteins AtfA and SOD1, in response to excess copper. (3) In the cytoplasm, copper is bound by copper chaperones that facilitate the delivery of copper to subcellular compartments (i.e., the Golgi complex) for storage or (4)(5 cytoplasmic and mitochondrial enzymes for functional activation. Parts 3, 4, and 5 are not proven in A. fumigatus, only in S. cerevisiae. ๑ When the concentration of copper in the cytoplasm exceeds the homeostatic capacity, A. fumigatus employs efflux to detoxify excess copper. Abbreviations: SOD, superoxide dismutase; ROS, reactive oxygen species. 
However, deletion of the $\operatorname{ctr} A 2$ or $\operatorname{ctr} C$ gene did not affect the concentration of copper in the cells, which may be explained by the redundancy of these two genes. By contrast, double deletion of the $\operatorname{ctr} A 2$ and $\operatorname{ctr} C$ genes dramatically reduced the intracellular copper content and resulted in growth and sporulation defects in copper-deficient medium (Park et al., 2014; Cai et al., 2017). Thus, CtrA2 and CtrC might play important roles in the regulation of copper homeostasis, growth, and sporulation of A. fumigatus in low-copper environments.

Although CtrA1 and CtrB exhibit homology with Ctr family members in $S$. cerevisiae, their exact function is unknown in A. fumigatus. Recent research demonstrated that deletion of the $\operatorname{ctr} B$ gene caused severe defects in hyphal growth in highor low-copper environments, suggesting that CtrB affects the growth and morphology of $A$. fumigatus independently of copper homeostasis (Cai et al., 2017). Interestingly, the colony defects of the $\triangle c t r B$ mutant could be suppressed by loss of the $\operatorname{ctr} A 2$ or $\operatorname{ctr} C$ gene. Moreover, the high copper sensitivity phenotype of the $\triangle c t r A 2$ mutant could also be partially recovered by deletion of the $c t r B$ gene (Cai et al., 2017). These results suggest that $\mathrm{Ctr} A 2, \mathrm{CtrC}$, and $\mathrm{CtrB}$ coordinately function to regulate hyphal growth and copper homeostasis, implying that Ctr transporters play a more complicated role in A. fumigatus than in yeast. To date, CtrA1 function in copper transport has not been revealed. Further studies are required to determine the nature of copper transport by CtrA1 in A. fumigatus.

\section{Copper Transporters Are Involved in the Efflux or Storage of Cytoplasmic Copper}

It has been reported that P-type ATPases are important regulators of intracellular copper levels, and the recently identified P-type ATPase, CrpA, is the major determinant of copper resistance in A. fumigatus (Wiemann et al., 2017). In contrast to Ctr family importers, CrpA has been identified as a copper exporter (Figure 1), and A. fumigatus mainly uses CrpA to remove excess copper. CrpA mainly localizes to the cellular surface, and it is involved in the transport of copper from the intracellular milieu to the extracellular environment. Deletion of the $\operatorname{crpA}$ gene caused significant intracellular copper accumulation and a copper-sensitive growth phenotype (Wiemann et al., 2017). Another P-type ATPase copper transporter, Ccc2, is also involved in copper homeostasis in S. cerevisiae (Fu et al., 1995). A. fumigatus also contains a potential homolog of $S$. cerevisiae Ccc2, designated CtpA (Figure 1). Similar to Ccc2 localization, CtpA is also localized on the Golgi complex in A. fumigatus (Upadhyay et al., 2013). Disruption of the ctpA gene leads to defective conidial pigmentation, which can be remediated by the addition of copper (Upadhyay et al., 2013). However, the role of CtpA as an exporter is yet to be clarified, and additional studies are required to determine the nature of copper transported by CtpA in A. fumigatus.

Cytochrome $c$ oxidase (COX), the terminal enzyme of the electron transport chain in mitochondria, requires copper as a cofactor (Tsukihara et al., 1996). Accordingly, it is important to import copper into the mitochondrial matrix for the eventual assembly of COX. In S. cerevisiae, Pic2, a mitochondrial carrier family protein, mediates copper import into the mitochondrial matrix (Vest et al., 2013). Moreover, Pic2 is a conserved protein in Aspergillus species, but a role for Pic2 as a copper transporter in mitochondria has not been revealed in these species.

\section{Copper Chaperones Are Involved in Copper Trafficking}

Because the copper buffering pool sequesters free copper in the cell, this copper then needs to be directed to the desired compartments. For this purpose, fungi have evolved highly specific and dedicated chaperones to direct copper trafficking. Currently, there are three copper transporting cytosolic chaperones (Atx1, Ccs1, and Cox17) in yeast (Smith et al., 2017). Atx1 is the copper chaperone responsible for delivering copper to the Golgi complex via Ccc2, whereas Ccs1 serves as the copper donor for superoxide dismutases (SODs). It has been demonstrated that Cox17 is responsible for transporting copper into mitochondria for the eventual assembly of COX. Although the chaperones Atx1, Ccs1, and Cox17 were only demonstrated in yeast, homologs of these three proteins are encoded in the genome of A. fumigatus (AFUB_008300, AFUB_025550, and AFUB_041410, respectively), but their function as chaperones is yet to be confirmed. Thus, further studies are required to determine their function in copper trafficking in A. fumigatus.

\section{Copper-Responsive Transcription Factors Are Involved in Copper Homeostasis}

Copper homeostasis in fungi is primarily regulated at the level of transcription through copper-responsive transcription factors. Most fungi carry two copper-responsive transcription factors to respond to copper starvation or toxicity. Currently, much can be learned regarding the copper-responsive transcription factors in A. fumigatus through examination of more wellstudied systems in yeast, and the copper status-specific transcription factors in A. fumigatus are similar to those found in S. cerevisiae.

In $S$. cerevisiae, copper starvation is sensed by the copperresponsive transcription factor Mac1, which activates Ctr1 and Ctr3 in response to low-copper environments (Jungmann et al., 1993; Smith et al., 2017). A. fumigatus also carries the S. cerevisiae Mac1 homolog AfMac1, the N-terminus of which contains the structure of conserved RGHR and GRP motifs for DNA binding. In addition, Cys-rich motifs are widely found at the C-terminus, which are involved in sensing copper (Cai et al., 2017; Kusuya et al., 2017). When A. fumigatus encounters low-copper concentrations, AfMacl induces the $\operatorname{ctr} C$ and $c t r A 2$ genes. An additional EMSA result demonstrated that AfMac1 directly binds to a copper response element in the promoter regions of the $\operatorname{ctr} A 2$ and $\operatorname{ctr} C$ genes with a defined consensus DNA motif (5'-TGTGCTCA-3') (Park et al., 2017), which is strikingly similar to the Macl-binding motif in S. cerevisiae (Jamison McDaniels et al., 1999; Keller et al., 2000), suggesting that the mechanism of Mac1-mediated copper homeostasis may be conserved across fungal species. 
Despite the important roles of copper in fungal biology, excess copper is potentially toxic to fungal cells. Thus, many fungi also carry a second copper-responsive transcription factor to respond to excess copper. In S. cerevisiae, excess copper is sensed by the copper-responsive transcription factor Ace1, which activates expression of metallothionein (MT) genes as well as the copperzinc SOD gene, in response to excess copper (Thiele, 1988; Gralla et al., 1991; Culotta et al., 1994). A. fumigatus also carries the S. cerevisiae Ace1 homolog AfAceA, and phylogenetic analyses revealed that most Ace1 homologs in fungi possess a conserved N-terminal signature including a zinc module and a copperregulatory domain, suggesting that excess copper-responsive transcription factors might have a common mechanism of DNA binding in fungi (Cai et al., 2018). When A. fumigatus cells encounter high copper concentrations, AfAceA induces expression of the copper extrusion pump CrpA, as well as ROS detoxification proteins AtfA and SOD1 (Wiemann et al., 2017). Although AfAceA also activates expression of the metallothionein CrdA to respond to excess copper, A. fumigatus utilizes the copper export protein CrpA rather than the copper metallothionein CrdA as a primary copper resistance mechanism (Wiemann et al., 2017). Therefore, A. fumigatus may use a different mechanism to that of $S$. cerevisiae to manage copper toxicity, which mainly uses metallothioneins to detoxify excess copper. S. cerevisiae Ace1 recognizes a specific copper response element in target gene promoters with the consensus sequence $5^{\prime}-\mathrm{TC}(\mathrm{T})_{4{ }_{6}} \mathrm{GCTG}-3^{\prime}$ (Furst et al., 1988; Huibregtse et al., 1989; Smith et al., 2017). However, we currently have a limited understanding of the AfAceA-binding motif in the target genes in A. fumigatus. Thus, further studies are needed to determine the AfAceA-binding motif in A. fumigatus, which will be one of the foci of future studies on the involvement of AfAceA in copper homeostasis regulation.

\section{THE COPPER HOMEOSTASIS SYSTEM IN OTHER ASPERGILLI}

Recent studies have illustrated the copper homeostasis systems in other aspergilli. For example, there are two key genes involved in regulating copper homeostasis in Aspergillus nidulans (Antsotegi-Uskola et al., 2017). First, the $\operatorname{crp} A$ gene, encoding a P-type ATPase, is involved in the transport of copper from the intracellular milieu to the extracellular environment. In addition, CrpA expression is highly inducible and dynamic in response to prolonged copper exposure. Second, the aceA gene, encoding a transcription factor, is necessary for the copper-inducible expression of CrpA. Another Aspergillus species, Aspergillus flavus, possesses two copies of the copper exporter, CrpA and $\mathrm{CrpB}$, which result in greater tolerance of copper in this species than in other aspergilli. Because of the redundant function of $\mathrm{CrpA}$ and $\mathrm{CrpB}$ proteins, only deletion of both genes resulted in extreme copper sensitivity. Similar to A. fumigatus and A. nidulans, A. flavus possess the same copper export machinery to prevent copper toxicity. In A. flavus, the $\mathrm{Cu}$-fist binding transcription factor AceA senses high levels of copper, and induces the P-type ATPases CrpA and $\mathrm{CrpB}$ as a detoxification mechanism (Yang et al., 2018).

\section{COPPER AND A. FUMIGATUS PATHOGENESIS}

\section{Copper-Based Strategies Deployed by the Host to Inhibit $A$. fumigatus Growth}

ROS production and transition metal homeostasis (mainly iron and zinc) are the major strategies employed by host immune cells to kill A. fumigatus (Dagenais and Keller, 2009; Lanternier et al., 2013; Heinekamp et al., 2015; Clark et al., 2016; Kasahara et al., 2016). Currently, accumulating evidence suggests that hosts have also evolved an antimicrobial strategy based on copper poisoning of microbes enclosed in macrophages (Wiemann et al., 2017). Recent research demonstrated that copper and ROS within macrophages have a close relationship in the killing of A. fumigatus (Wiemann et al., 2017; Yang et al., 2018).

Although the precise mechanisms of copper mobilization in macrophages remain to be fully explored, some studies revealed that macrophages actively accumulate and compartmentalize copper by increasing the expression of the high-affinity copper transporter Ctr1 at the plasma membrane and translocating the P-type copper ATPase pump ATP7A to the phagolysosomal membrane (White et al., 2009; Achard et al., 2012; Ding et al., 2013; Garcia-Santamarina and Thiele, 2015). Because the host copper transport response to different microbial pathogens is generally conserved, macrophages encountering A. fumigatus spores also react by upregulating Ctr1 and aggregating ATP7A at the phagolysosomal membrane (Figure 2; Wiemann et al., 2017). When copper is transported into macrophages, it can potentiate redox potential and form highly-reactive hydroxyl radicals, thereby potentiating hostderived ROS toxicity (Figure 2; Thor et al., 1982; White and Clark, 1988). Therefore, host-derived ROS and copper responses could not be clearly separated in A. fumigatus, and copper mobilized by macrophages exerts its lethality by potentiating host-derived ROS toxicity.

\section{Copper Homeostasis and Virulence}

Because host innate immune cells use copper compartmentalization as a means to exploit copper toxicity and enhance microbial killing, fungal pathogens possess highly orchestrated mechanisms to regulate copper homeostasis for preventing copper toxicity, thereby facilitating their survival. As mentioned above, $A$. fumigatus utilizes several protein classes to regulate copper homeostasis, including copper transporters (import and export) and copper-binding transcription factors (AfAceA and AfMac1), and these proteins play an important role in $A$. fumigatus virulence. Although the copper importers CtrA2 and CtrC failed to affect the mouse survival rate (Park et al., 2014), copper exporter CrpA is critical for A. fumigatus virulence as the $\triangle \operatorname{crp} A$ mutant showed significantly decreased virulence in an immunocompromised murine model of invasive aspergillosis (IA) (Wiemann et al., 2017). Because $c r p A$ was highly induced by copper in an AfAceA-dependent manner, AfAceA is also considered a virulence factor for $A$. fumigatus. In the non-neutropenic IA murine model, the $\triangle A f A c e A$ mutant was significantly less virulent than the corresponding wild-type 


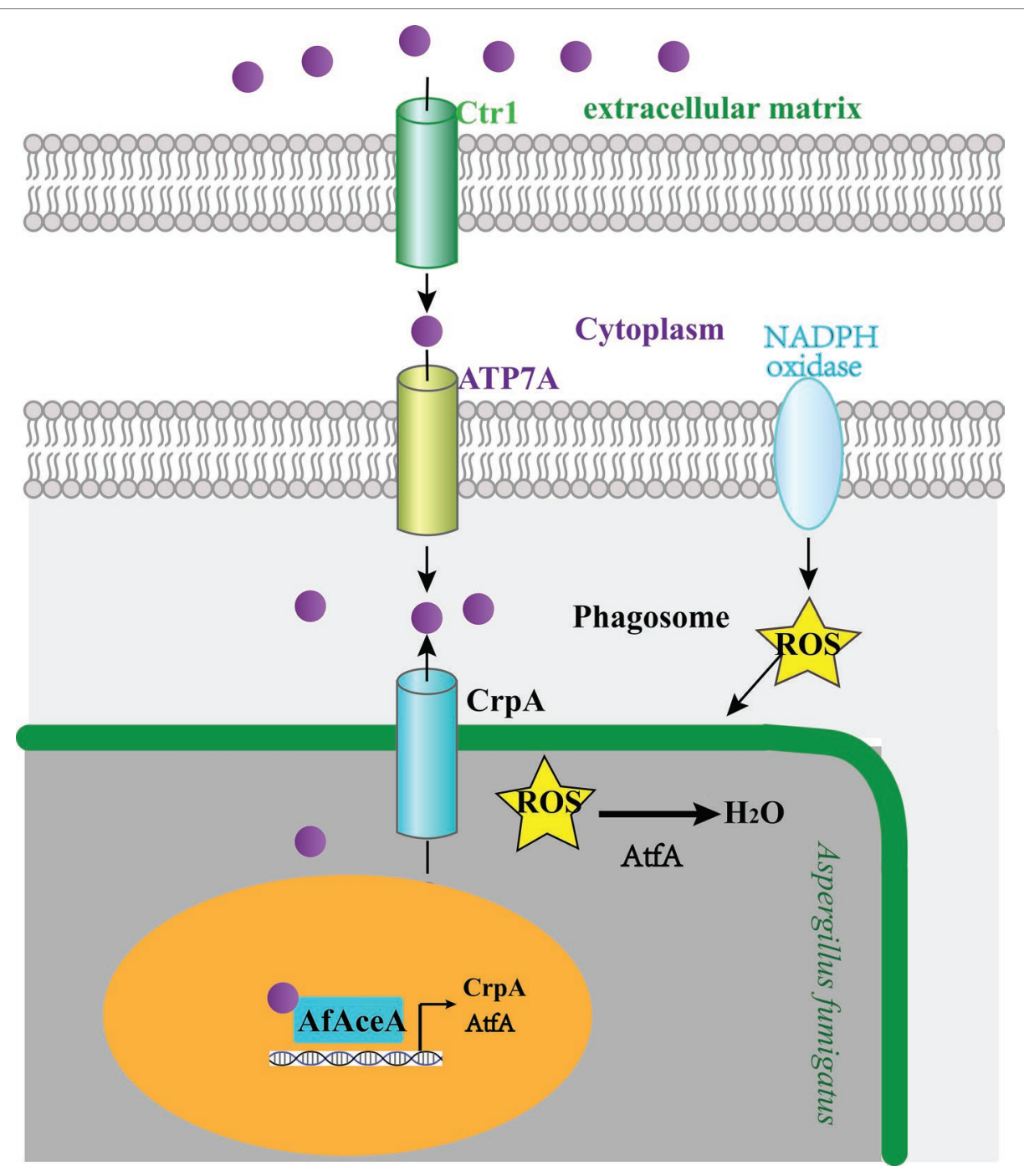

FIGURE 2 | A. fumigatus response to host-imposed copper toxicity. Macrophages encountering A. fumigatus react by upregulating the host copper (purple spheres) transporter Ctr1 and aggregating ATP7A at the phagolysosomal membrane to facilitate copper uptake. A. fumigatus AfAceA utilizes the copper exporter CrpA and the ROS-responsive transcription factor AtfA to respond to host-imposed copper toxicity.

(Wiemann et al., 2017). Similar to AfAceA, another transcription factor, designated AfMac1, is also necessary for A. fumigatus virulence as deletion of Afmacl significantly reduced virulence (Cai et al., 2017; Park et al., 2017).

\section{A. fumigatus Response to Host-Imposed Copper Toxicity}

To survive host-imposed copper toxicity, A. fumigatus has evolved some effective mechanisms to detoxify copper (Wiemann et al., 2017). A. fumigatus utilizes copper export as a primary copper resistance mechanism, and employs AfAceA to induce the copper exporter CrpA to prevent copper toxicity (Wiemann et al., 2017). In addition to copper export, A. fumigatus also activates AfAceA to induce the metallothionein CrdA as an intracellular copper buffer. Deletion of the $c r d A$ gene does not affect copper tolerance, whereas in AfAceA or crpA mutants, overexpression of $c r d A$ rescues copper sensitivity (Cai et al., 2018), thereby serving as a secondary mechanism of preventing host-imposed copper toxicity.

Macrophages generate ROS upon infection through the activity of the NADPH oxidase, which is an effective strategy for killing A. fumigatus. When copper is mobilized into macrophages, it can potentiate the redox potential and thereby oxidize lipids, nucleic acids, and proteins; these macromolecular insults have long been considered the primary basis for copper cytotoxicity (Garcia-Santamarina and Thiele, 2015). Taken together, copper mobilized by host cells partially exerts its lethality by potentiating host ROS toxicity. Thus, an efficient ROS detoxification system is critical for the survival of A. fumigatus within this hostile environment. A. fumigatus employs AfAceA to induce the ROS-responsive transcription factor AtfA, which is suggested to specifically govern spore ROS defense to cope with ROS stress within macrophages, and deletion of the atfA gene results in decreased survival in response to challenge with macrophages 
(Wiemann et al., 2017). Taken together, A. fumigatus AfAceA utilizes the copper exporter CrpA and the ROS-responsive transcription factor AtfA as the primary host countermeasures to host-imposed copper toxicity (Figure 2).

\section{Copper-Dependent Enzymes Involved in A. fumigatus Virulence}

Melanin is a phenolic polymer and a well-known virulence factor (Langfelder et al., 2003; Nosanchuk and Casadevall, 2006). For example, melanin enhances fungal attachment to host tissues, absorbs host-generated ROS, and helps A. fumigatus evade host immune recognition (Thywissen et al., 2011; Bayry et al., 2014). Recent research has also demonstrated that A. fumigatus melanin can block LC3-associated phagocytosis to promote pathogenicity (Akoumianaki et al., 2016). Thus, A. fumigatus melanin is strongly associated with fungal virulence, and mutants defective in melanization are less virulent. In A. fumigatus, melanin synthesis requires the copper-dependent laccases Abr1 and Abr2, and deletion of either abr1 or $a b r 2$ significantly reduces melanin formation, making $A$. fumigatus spores more sensitive to destruction by macrophages (Pihet et al., 2009). As laccases require copper as their cofactor, dysregulation of copper homeostasis might affect laccase activity and/or expression (Upadhyay et al., 2013). Consistent with this conclusion, previous research found that the copper transporter CtpA is involved in copper homeostasis and that it plays an important role in supplying copper to laccases Abr1/2; thus, $\operatorname{ctp} A$ disruption leads to defective conidial pigmentation (Upadhyay et al., 2013).

In A. fumigatus, SODs are the most important family of enzymes for ROS detoxification, and the A. fumigatus genome encodes four putative SODs (AfSOD1-4). Among these four SODs, cytoplasmic $\mathrm{Cu} / \mathrm{ZnSOD} 1$ and mitochondrial MnSOD2 play major roles in detoxifying ROS (Lambou et al., 2010). However, A. fumigatus SODs are not involved in fungal virulence, whereas SODs are required for virulence in Cryptococcus neoformans and Candida albicans (Cox et al., 2003; Narasipura et al., 2005). A reasonable explanation may be that A. fumigatus SODs are intracellular and therefore do not neutralize extracellular ROS produced by innate immune cells, which is different from the mechanism in C. neoformans and C. albicans. Accordingly, other ROS-scavenging pathways may play major roles in counteracting ROS in A. fumigatus. In conclusion, the copperdependent enzyme laccase is closely related to A. fumigatus pathogenesis; however, $\mathrm{Cu} / \mathrm{ZnSODs}$ are not apparently required for pathogenesis.

\section{POTENTIAL FOR COPPER HOMEOSTASIS AS A NOVEL ANTIFUNGAL THERAPEUTIC TARGET}

Currently, the classic antifungal drugs used to treat fungal pathogens do not rapidly inhibit $A$. fumigatus growth, and hence, mortality rates remain unacceptably high (Xie et al., 2014). Thus, a major current challenge is developing new antifungal drugs that target $A$. fumigatus-specific metabolic pathways opposed to those targeted by the classic antifungal drugs. As previously described, host cells can inhibit microbial growth by intoxicating fungal pathogens with excess copper (Ding et al., 2013; Wiemann et al., 2017). Accordingly, one strategy could be therapeutically inhibiting the growth of fungal pathogens in the host, and the regulators of copper homeostasis have emerged as ideal targets for the development of novel antifungal therapies.

Because A. fumigatus has evolved efficient methods to counter copper-mediated killing though copper export (Wiemann et al., 2017), we propose that a therapeutic approach based on preventing copper extrusion would be deleterious for $A$. fumigatus cells. As the extrusion of copper is mainly mediated by the copper exporter CrpA in A. fumigatus, we speculate that CrpA could serve as a drug target, and any new compounds that interfere with the function of CrpA would predictably inhibit A. fumigatus growth within host tissues. In addition, CrpA has cysteine-rich copper-binding motifs (MBD) in its N-terminus, differing from the human homolog, and hence, any compound inhibiting the function of CrpA by targeting its N-terminus without affecting the human homolog would be theoretically valid. In A. fumigatus, the copper-responsive transcription factor AfAceA is the most important modulator of copper homeostasis and virulence, and A. fumigatus employs AfAceA to activate the copper exporter CrpA and ROS-degrading proteins to prevent copper toxicity. (Wiemann et al., 2017). Notably, the $\triangle \operatorname{crp} A$ mutant showed decreased virulence similar to that of the $\triangle A f A c e A$ mutant in an immunocompromised murine model of IA, suggesting that the copper exporter plays a major role in A. fumigatus virulence. P-type ATPases are considered therapeutic targets due to their accessibility on cell membranes, coupled with the recent progress in specifically targeting P-type ATPases (Kirk, 2015; Turner, 2016). Inactivation of the P-type ATPase CrpA alone is sufficient to decrease virulence, and efforts to target CrpA may hold promise for future work. In summary, regulators of copper homeostasis have emerged as ideal targets for developing novel antifungal therapies. Future work should aim to discover and develop new drugs that specifically block the regulation of copper homeostasis.

\section{Copper lonophores Could Potentially Serve As Antifungal Agents}

Because regulators of copper homeostasis can be regarded as therapeutic targets, molecules interfering with copper homeostasis could be used as antifungal drugs. Copper ionophores can coordinate and shuttle copper from the extracellular environment to the intracellular milieu (Garcia-Santamarina and Thiele, 2015), thus, some studies found that copper ionophores can potentially serve as antifungal agents. For example, zinc pyrithione (ZPT), an antimicrobial agent widely used in antidandruff shampoos, can act as a copper ionophore (Chen and Hill, 2005; Reeder et al., 2011). However, ZPT is only useful in antidandruff shampoos due to toxic. The mechanism of action of ZPT as an antifungal agent has been investigated in S. cerevisiae, with data indicating that ZPT acts by dramatically increasing cellular copper concentrations to inhibit the growth 
of S. cerevisiae (Reeder et al., 2011). As observed in S. cerevisiae, ZPT also increases copper concentrations to inhibit the growth of Malassezia globosa, the most common fungus known to be associated with dandruff (Reeder et al., 2011).

Recently, boronic ester-masked 8-hydroxyquinoline derivative (QBP), which is a nontoxic protected form of the well-characterized copper ionophore 8-hydroxyquinoline (8HQ), has been used as an antifungal agent to inhibit the growth of $C$. neoformans in the lungs. The oxidative burst generated by activated macrophages mediates the conversion of $\mathrm{QBP}$ to $8 \mathrm{HQ}$, which subsequently translocates copper into fungi within the phagolysosome, eliciting copper-dependent cytotoxicity (Festa et al., 2014). Therefore, the conditional activation of copper ionophores represents a promising approach to treating systemic fungal infections. Because 8HQ has broad-spectrum antimicrobial activity, QBP may potentially have a broad range of applications. Thus, future works are needed to apply this strategy to the treatment of A. fumigatus infection.

As mentioned above, copper ionophores are considered to be antifungal agents, mainly because they can increase the concentration of copper in cells. Thereby, compounds that inhibit the expression of copper detoxification genes and thus increase the intracellular copper concentration can also be considered as antifungal agents. AfAceA and CrpA are key proteins for copper detoxification and virulence in A. fumigatus. Thus, pharmacologic disruption of AfAceA or CrpA function would be predicted to induce fungal death due to the increased copper concentration in cells. Accordingly, AfAceA or CrpA could also be used as drug targets for the inhibitory effect of copper ionophores on fungal growth. Moreover, copper ionophores together with AfAceA or CrpA inhibitors could potentially magnify the copper-mediated toxicity.

\section{FUTURE DIRECTIONS AND CONCLUSIONS}

Because the expression of copper tolerance genes is critical for the survival and virulence of fungal pathogens, pharmacological disruption of the function of copper tolerance genes would be predicted to have the following beneficial effects: (1) Induce fungal death: the loss of copper tolerance genes would lead to toxic levels of copper that would be expected to have fungistatic and/or fungicidal effects on the organism. (2) Enhance the activity of current antifungal drugs: the clinical efficacy of current antifungal drugs may be compromised by intrinsic or acquired resistance. Combination antifungal therapy with agents of different mechanistic classes could promote fungal killing and clinical efficacy and provide an alternative to

\section{REFERENCES}

Achard, M. E., Stafford, S. L., Bokil, N. J., Chartres, J., Bernhardt, P. V., Schembri, M. A., et al. (2012). Copper redistribution in murine macrophages in response to Salmonella infection. Biochem. J. 444, 51-57. doi: 10.1042/ BJ20112180

Akoumianaki, T., Kyrmizi, I., Valsecchi, I., Gresnigt, M. S., Samonis, G., Drakos, E., et al. (2016). Aspergillus cell wall melanin blocks LC3-associated monotherapy regimens. This suggests that combining inhibitors of copper tolerance proteins with one or more current antifungals could increase efficacy, promote fungal killing, and prevent the emergence of drug resistance more effectively than monotherapy regimens. In particular, combination therapy might reduce the hepatotoxicity caused by long-term exposure to high doses of azoles drugs. Thus, the AfAceA and $\operatorname{crpA}$ copper tolerance genes may be potential drug targets, although deletion of these genes in A. fumigatus leads only to attenuated virulence, not avirulence. Taken together, the regulation of copper homeostasis provides promising therapeutic targets for combating A. fumigatus infection. Furthermore, by targeting copper homeostasis with a specific combination of two drugs, the possibility that the fungus will develop mutations that increase drug resistance is significantly lessened. Thus, future efforts should be directed to the discovery of new compounds that can specifically block the function of the proteins that regulate copper homeostasis. Because mammalian hosts can kill $A$. fumigatus by accumulating copper within the phagolysosome of macrophages, it is important to understand the underlying mechanisms of copper mobilization within macrophages and the corresponding fungal responses to excess copper. Biochemical approaches to manipulate copper homeostasis may provide promising strategies for the development of novel antifungal therapies.

\section{AUTHOR CONTRIBUTIONS}

JS conducted the literature study and wrote the manuscript. $\mathrm{RL}$ and JJ edited and revised the manuscript.

\section{FUNDING}

This work was financially supported by the National Natural Science Foundation of China (NSFC31800058 to JS, NSFC31770613 to JJ and NSFC81703569, 81870005 to RL), the Natural Science Foundation of the Jiangsu Higher Education Institutions of China (17KJB180004 to JS and 18KJA180003 to RL), a grant from the Natural Science Foundation by Jiangsu Normal University (17XLR020 to JS), and the Project of Xuzhou Applied and Basic Research (KC18009 to RL). The National Natural Science Foundation of China: Funding researchers for scientific research in China. The Natural Science Foundation by Jiangsu Normal University: Funding young teachers from colleges and universities in Jiangsu Province for scientific research. The Project of Xuzhou Applied and Basic Research: Promote Xuzhou Applied and Basic Research. 10.1016/j.chom.2015.12.002

Antsotegi-Uskola, M., Markina-Inarrairaegui, A., and Ugalde, U. (2017). Copper resistance in Aspergillus nidulans relies on the PI-Type ATPase CrpA, regulated by the transcription factor AceA. Front. Microbiol. 8:912. doi: 10.3389/ fmicb.2017.00912

Bayry, J., Beaussart, A., Dufrene, Y. F., Sharma, M., Bansal, K., Kniemeyer, O., et al. (2014). Surface structure characterization of Aspergillus fumigatus 
conidia mutated in the melanin synthesis pathway and their human cellular immune response. Infect. Immun. 82, 3141-3153. doi: 10.1128/Iai.01726-14

Brown, G. D., Denning, D. W., Gow, N. A. R., Levitz, S. M., Netea, M. G., and White, T. C. (2012a). Hidden killers: human fungal infections. Sci. Transl. Med. 4, 1-9. doi: 10.1126/scitranslmed.3004404

Brown, G. D., Denning, D. W., and Levitz, S. M. (2012b). Tackling human fungal infections. Science 336:647. doi: 10.1126/science.1222236

Cai, Z., Du, W., Zeng, Q., Long, N., Dai, C., and Lu, L. (2017). Cu-sensing transcription factor Mac1 coordinates with the Ctr transporter family to regulate $\mathrm{Cu}$ acquisition and virulence in Aspergillus fumigatus. Fungal Genet. Biol. 107, 31-43. doi: 10.1016/j.fgb.2017.08.003

Cai, Z. D., Du, W. L., Zhang, Z., Guan, L. Y., Zeng, Q. Q., Chai, Y. F., et al. (2018). The Aspergillus fumigatus transcription factor AceA is involved not only in $\mathrm{Cu}$ but also in $\mathrm{Zn}$ detoxification through regulating transporters CrpA and ZrcA. Cell. Microbiol. 20. doi: 10.1111/cmi.12864

Casey, A. L., Adams, D., Karpanen, T. J., Lambert, P. A., Cookson, B. D., Nightingale, P., et al. (2010). Role of copper in reducing hospital environment contamination. J. Hosp. Infect. 74, 72-77. doi: 10.1016/j.jhin.2009.08.018

Chen, T. A., and Hill, P. B. (2005). The biology of Malassezia organisms and their ability to induce immune responses and skin disease. Vet. Dermatol. 16, 4-26. doi: 10.1111/j.1365-3164.2005.00424.x

Clark, H. L., Jhingran, A., Sun, Y., Vareechon, C., Carrion, S. D., Skaar, E. P., et al. (2016). Zinc and manganese chelation by neutrophil S100A8/A9 (calprotectin) limits extracellular Aspergillus fumigatus hyphal growth and corneal infection. J. Immunol. 196, 336-344. doi: 10.4049/jimmunol.1502037

Cox, G. M., Harrison, T. S., McDade, H. C., Taborda, C. P., Heinrich, G., Casadevall, A., et al. (2003). Superoxide dismutase influences the virulence of Cryptococcus neoformans by affecting growth within macrophages. Infect. Immun. 71, 173-180. doi: 10.1128/Iai.71.1.173-180.2003

Culotta, V. C., Howard, W. R., and Liu, X. F. (1994). CRS5 encodes a metallothioneinlike protein in Saccharomyces cerevisiae. J. Biol. Chem. 269, 25295-25302.

Dagenais, T. R. T., and Keller, N. P. (2009). Pathogenesis of Aspergillus fumigatus in invasive Aspergillosis. Clin. Microbiol. Rev. 22, 447-465. doi: 10.1128/ Cmr.00055-08

Dancis, A., Haile, D., Yuan, D. S., and Klausner, R. D. (1994). The Saccharomyces cerevisiae copper transport protein (Ctrlp). Biochemical characterization, regulation by copper, and physiologic role in copper uptake. J. Biol. Chem. 269, 25660-25667.

Ding, C., Festa, R. A., Chen, Y. L., Espart, A., Palacios, O., Espin, J., et al. (2013). Cryptococcus neoformans copper detoxification machinery is critical for fungal virulence. Cell Host Microbe 13, 265-276. doi: 10.1016/j.chom.2013.02.002

Festa, R. A., Helsel, M. E., Franz, K. J., and Thiele, D. J. (2014). Exploiting innate immune cell activation of a copper-dependent antimicrobial agent during infection. Chem. Biol. 21, 977-987. doi: 10.1016/j. chembiol.2014.06.009

Fridovich, I. (1983). Superoxide radical: an endogenous toxicant. Annu. Rev. Pharmacol. Toxicol. 23, 239-257. doi: 10.1146/annurev.pa.23.040183.001323

Fu, D. D., Beeler, T. J., and Dunn, T. M. (1995). Sequence, mapping and disruption of $\mathrm{Ccc} 2$, a gene that cross-complements the $\mathrm{Ca}^{2+}$-sensitive phenotype of Csg1 mutants and encodes a P-Type Atpase belonging to the $\mathrm{Cu}^{2+}$-Atpase subfamily. Yeast 11, 283-292. doi: 10.1002/yea.320110310

Furst, P., Hu, S., Hackett, R., and Hamer, D. (1988). Copper activates metallothionein gene transcription by altering the conformation of a specific DNA binding protein. Cell 55, 705-717. doi: 10.1016/0092-8674(88)90229-2

Garcia-Santamarina, S., and Thiele, D. J. (2015). Copper at the fungal pathogenhost axis. J. Biol. Chem. 290, 18945-18953. doi: 10.1074/jbc.R115.649129

Gralla, E. B., Thiele, D. J., Silar, P., and Valentine, J. S. (1991). ACE1, a copperdependent transcription factor, activates expression of the yeast copper, zinc superoxide dismutase gene. Proc. Natl. Acad. Sci. USA 88, 8558-8562.

Hassett, R., Dix, D. R., Eide, D. J., and Kosman, D. J. (2000). The Fe(II) permease Fet $4 p$ functions as a low affinity copper transporter and supports normal copper trafficking in Saccharomyces cerevisiae. Biochem. J. 351, 477-484. doi: 10.1042/0264-6021:3510477

Heinekamp, T., Schmidt, H., Lapp, K., Pahtz, V., Shopova, I., Koster-Eiserfunke, N., et al. (2015). Interference of Aspergillus fumigatus with the immune response. Semin. Immunopathol. 37, 141-152. doi: 10.1007/s00281-014-0465-1

Huibregtse, J. M., Engelke, D. R., and Thiele, D. J. (1989). Copper-induced binding of cellular factors to yeast metallothionein upstream activation sequences. Proc. Natl. Acad. Sci. USA 86, 65-69.
Jamison McDaniels, C. P., Jensen, L. T., Srinivasan, C., Winge, D. R., and Tullius, T. D. (1999). The yeast transcription factor Mac1 binds to DNA in a modular fashion. J. Biol. Chem. 274, 26962-26967. doi: 10.1074/jbc.274.38.26962 Jungmann, J., Reins, H. A., Lee, J. W., Romeo, A., Hassett, R., Kosman, D., et al. (1993). Mac1, a nuclear regulatory protein related to $\mathrm{Cu}$-dependent transcription factors is involved in $\mathrm{Cu} / \mathrm{Fe}$ utilization and stress resistance in yeast. $E M B O J .12,5051-5056$. doi: 10.1002/j.1460-2075.1993.tb06198.x

Kasahara, S., Jhingran, A., Dhingra, S., Salem, A., Cramer, R. A., and Hohl, T. M. (2016). Role of granulocyte-macrophage colony-stimulating factor signaling in regulating neutrophil antifungal activity and the oxidative burst during respiratory fungal challenge. J. Infect. Dis. 213, 1289-1298. doi: 10.1093/ infdis/jiw054

Keller, G., Gross, C., Kelleher, M., and Winge, D. R. (2000). Functional independence of the two cysteine-rich activation domains in the yeast Macl transcription factor. J. Biol. Chem. 275, 29193-29199. doi: 10.1074/jbc. M001552200

Kirk, K. (2015). Ion regulation in the malaria parasite. Annu. Rev. Microbiol. 71, 341-359. doi: 10.1146/annurev-micro-091014-104506

Kusuya, Y., Hagiwara, D., Sakai, K., Yaguchi, T., Gonoi, T., and Takahashi, H. (2017). Transcription factor Afmacl controls copper import machinery in Aspergillus fumigatus. Curr. Genet. 63, 777-789. doi: 10.1007/ s00294-017-0681-z

Kwon-Chung, K. J., and Sugui, J. A. (2013). Aspergillus fumigatus-what makes the species a ubiquitous human fungal pathogen? PLoS Pathog. 9:e1003743. doi: 10.1371/journal.ppat.1003743

Lambou, K., Lamarre, C., Beau, R., Dufour, N., and Latge, J. P. (2010). Functional analysis of the superoxide dismutase family in Aspergillus fumigatus. Mol. Microbiol. 75, 910-923. doi: 10.1111/j.1365-2958.2009.07024.x

Langfelder, K., Streibel, M., Jahn, B., Haase, G., and Brakhage, A. A. (2003). Biosynthesis of fungal melanins and their importance for human pathogenic fungi. Fungal Genet. Biol. 38, 143-158. doi: 10.1016/S1087-1845(02)00526-1

Lanternier, F., Cypowyj, S., Picard, C., Bustamante, J., Lortholary, O., Casanova, J. L., et al. (2013). Primary immunodeficiencies underlying fungal infections. Curr. Opin. Pediatr. 25, 736-747. doi: 10.1097/MOP.0000000000000031

Lazary, A., Weinberg, I., Vatine, J. J., Jefidoff, A., Bardenstein, R., Borkow, G., et al. (2014). Reduction of healthcare-associated infections in a long-term care brain injury ward by replacing regular linens with biocidal copper oxide impregnated linens. Int. J. Infect. Dis. 24, 23-29. doi: 10.1016/j. ijid.2014.01.022

Macomber, L., and Imlay, J. A. (2009). The iron-sulfur clusters of dehydratases are primary intracellular targets of copper toxicity. Proc. Natl. Acad. Sci. USA 106, 8344-8349. doi: 10.1073/pnas.0812808106

Narasipura, S. D., Chaturvedi, V., and Chaturvedi, S. (2005). Characterization of Cryptococcus neoformans variety gattii SOD2 reveals distinct roles of the two superoxide dismutases in fungal biology and virulence. Mol. Microbiol. 55, 1782-1800. doi: 10.1111/j.1365-2958.2005.04503.x

Nevitt, T., Ohrvik, H., and Thiele, D. J. (2012). Charting the travels of copper in eukaryotes from yeast to mammals. Biochim. Biophys. Acta 1823, 1580-1593. doi: 10.1016/j.bbamcr.2012.02.011

Nosanchuk, J. D., and Casadevall, A. (2006). Impact of melanin on microbial virulence and clinical resistance to antimicrobial compounds. Antimicrob. Agents Chemother. 50, 3519-3528. doi: 10.1128/AAC.00545-06

Park, Y. S., Kang, S., Seo, H., and Yun, C. W. (2018). A copper transcription factor, AfMac1 regulates both iron and copper homeostasis in the opportunistic fungal pathogen Aspergillus fumigatus. Biochem. J. 475, 2831-2845. doi: 10.1042/Bcj20180399

Park, Y. S., Kim, T. H., and Yun, C. W. (2017). Functional characterization of the copper transcription factor AfMacl from Aspergillus fumigatus. Biochem. J. 474, 2365-2378. doi: 10.1042/BCJ20170191

Park, Y. S., Lian, H., Chang, M., Kang, C. M., and Yun, C. W. (2014). Identification of high-affinity copper transporters in Aspergillus fumigatus. Fungal Genet. Biol. 73, 29-38. doi: 10.1016/j.fgb.2014.09.008

Pena, M. M., Puig, S., and Thiele, D. J. (2000). Characterization of the Saccharomyces cerevisiae high affinity copper transporter Ctr3. J. Biol. Chem. 275, 33244-33251. doi: 10.1074/jbc.M005392200

Pihet, M., Vandeputte, P., Tronchin, G., Renier, G., Saulnier, P., Georgeault, S., et al. (2009). Melanin is an essential component for the integrity of the cell wall of Aspergillus fumigatus conidia. BMC Microbiol. 9:177. doi: 10.1186/1471-2180-9-177 
Reeder, N. L., Kaplan, J., Xu, J., Youngquist, R. S., Wallace, J., Hu, P., et al. (2011). Zinc pyrithione inhibits yeast growth through copper influx and inactivation of iron-sulfur proteins. Antimicrob. Agents Chemother. 55, 5753-5760. doi: 10.1128/Aac.00724-11

Rees, E. M., and Thiele, D. J. (2007). Identification of a vacuole-associated metalloreductase and its role in Ctr2-mediated intracellular copper mobilization. J. Biol. Chem. 282, 21629-21638. doi: 10.1074/jbc.M703397200

Robbins, N., Caplan, T., and Cowen, L. E. (2017). Molecular evolution of antifungal drug resistance. Annu. Rev. Microbiol. 71, 753-775. doi: 10.1146/ annurev-micro-030117-020345

Shapiro, R. S., Robbins, N., and Cowen, L. E. (2011). Regulatory circuitry governing fungal development, drug resistance, and disease. Microbiol. Mol. Biol. $R$ 75, 213-267. doi: 10.1128/Mmbr.00045-10

Smith, A. D., Logeman, B. L., and Thiele, D. J. (2017). Copper acquisition and utilization in fungi. Annu. Rev. Microbiol. 71, 597-623. doi: 10.1146/ annurev-micro-030117-020444

Thiele, D. J. (1988). ACE1 regulates expression of the Saccharomyces cerevisiae metallothionein gene. Mol. Cell. Biol. 8, 2745-2752. doi: 10.1128/MCB.8.7.2745

Thor, H., Smith, M. T., Hartzell, P., Bellomo, G., Jewell, S. A., and Orrenius, S. (1982). The metabolism of menadione (2-methyl-1,4-naphthoquinone) by isolated hepatocytes. A study of the implications of oxidative stress in intact cells. J. Biol. Chem. 257, 12419-12425.

Thywissen, A., Heinekamp, T., Dahse, H. M., Schmaler-Ripcke, J., Nietzsche, S., Zipfel, P. F., et al. (2011). Conidial dihydroxynaphthalene melanin of the human pathogenic fungus Aspergillus fumigatus interferes with the host endocytosis pathway. Front. Microbiol. 2:96. doi: 10.3389/fmicb.2011.00096

Tsukihara, T., Aoyama, H., Yamashita, E., Tomizaki, T., Yamaguchi, H., ShinzawaItoh, K., et al. (1996). The whole structure of the 13-subunit oxidized cytochrome c oxidase at 2.8 angstrom. Science 272, 1136-1144. doi: 10.1126/ science. 272.5265 .1136

Turner, H. (2016). Spiroindolone NITD609 is a novel antimalarial drug that targets the P-type ATPase PfATP4. Future Med. Chem. 8, 227-238. doi: 10.4155/fmc.15.177

Upadhyay, S., Torres, G., and Lin, X. (2013). Laccases involved in 1,8-dihydroxynaphthalene melanin biosynthesis in Aspergillus fumigatus are regulated by developmental factors and copper homeostasis. Eukaryot. Cell 12, 1641-1652. doi: 10.1128/EC.00217-13

Vest, K. E., Leary, S. C., Winge, D. R., and Cobine, P. A. (2013). Copper import into the mitochondrial matrix in Saccharomyces cerevisiae is mediated by Pic2, a mitochondrial carrier family protein. J. Biol. Chem. 288, 23884-23892. doi: $10.1074 /$ jbc.M113.470674

White, E. J., and Clark, J. B. (1988). Menadione-treated synaptosomes as a model for post-ischaemic neuronal damage. Biochem. J. 253, 425-433. doi: 10.1042/bj2530425

White, C., Lee, J., Kambe, T., Fritsche, K., and Petris, M. J. (2009). A role for the ATP7A copper-transporting ATPase in macrophage bactericidal activity. J. Biol. Chem. 284, 33949-33956. doi: 10.1074/jbc.M109.070201

Wiemann, P., Perevitsky, A., Lim, F. Y., Shadkchan, Y., Knox, B. P., Landero Figueora, J. A., et al. (2017). Aspergillus fumigatus copper export machinery and reactive oxygen intermediate defense counter host copper-mediated oxidative antimicrobial offense. Cell Rep. 19, 1008-1021. doi: 10.1016/j. celrep.2017.04.019

Xie, J. L., Polvi, E. J., Shekhar-Guturja, T., and Cowen, L. E. (2014). Elucidating drug resistance in human fungal pathogens. Future Microbiol. 9, 523-542. doi: $10.2217 / \mathrm{fmb} .14 .18$

Yang, K., Shadkchan, Y., Tannous, J., Landero Figueroa, J. A., Wiemann, P., Osherov, N., et al. (2018). Contribution of ATPase copper transporters in animal but not plant virulence of the crossover pathogen Aspergillus flavus. Virulence 9, 1273-1286. doi: 10.1080/21505594.2018.1496774

Conflict of Interest Statement: The authors declare that the research was conducted in the absence of any commercial or financial relationships that could be construed as a potential conflict of interest.

Copyright (c) 2019 Song, Li and Jiang. This is an open-access article distributed under the terms of the Creative Commons Attribution License (CC BY). The use, distribution or reproduction in other forums is permitted, provided the original author(s) and the copyright owner(s) are credited and that the original publication in this journal is cited, in accordance with accepted academic practice. No use, distribution or reproduction is permitted which does not comply with these terms. 